\title{
Serous cystadenoma of the pancreas with atypical clinical manifestations: A case report
}

\author{
YUAN GAO, JING PING ZHANG and PING ZHU \\ Department of General Surgery, The Second People's Hospital of Changzhou Affiliated to Nanjing Medical University, \\ Changzhou, Jiangsu 213000, P.R. China
}

Received November 5, 2015; Accepted January 27, 2016

DOI: $10.3892 / \mathrm{mco} .2016 .771$

\begin{abstract}
The present study reported a $4.8 \mathrm{~cm}$ space-occupying cystic solid mass of the pancreas, which caused recurrent bilateral lower back discomfort in a 60-year-old female. Combined with the clinical data, an impression of a solid-pseudopapillary neoplasm was generated prior to surgery. Abdominal exploration revealed splenic vessel encasement by the mass similar to the invasion observed in a malignant tumor. Distal pancreatectomy with splenectomy was subsequently performed. Grossly, the tumor was solid and consisted of numerous small cysts. Histopathological examination of the cystic solid mass revealed classic microcystic serous cystadenoma of the pancreas. These findings suggested that microcystic serous cystadenoma can exhibit atypical clinical manifestations. Asymptomatic patients with a small lesion $(<4 \mathrm{~cm})$ require imaging surveillance every 2 years; however, tumors $>4 \mathrm{~cm}$ with atypical presentations require surgical resection.
\end{abstract}

\section{Introduction}

Serous cystadenoma of pancreas (SCA) is a benign epithelial neoplasm of uncertain origin. Immunohistochemical and ultrastructural studies have suggested that SCA may originate from centroacinar or intercalated duct cells. It can occur in any region of the pancreas, predominantly in older females (1). According to the literature, the majority of SCAs are small $(<1 \mathrm{~cm})$ and are identified incidentally. Grossly, SAC is a well-circumscribed mass within numerous small cysts ('honeycomb' appearance) on cut surface with a central fibrotic scar. Histologically, majority of SACs are 'microcystic' with numerous cysts exhibiting a diameter $<2 \mathrm{~cm}$ and rarely with calcification in the fibrous septa. Lesions with a diameter $>2 \mathrm{~cm}$ were defined as macrocystic serous cystadenoma $(2,3)$.

Correspondence to: Dr Ping Zhu, Department of General Surgery, The Second People's Hospital of Changzhou Affiliated to Nanjing Medical University, 29 Xinglong Alley Changzhou, Jiangsu 213000, P.R. China

E-mail: zhuping20153@sina.com

Key words: serous cystadenoma, pancreas, distal pancreatectomy, microcytic serous cystadenoma
The cystic fluid is watery clear or straw-colored without mucin production. The cysts are lined by low cuboidal cells with clear to eosinophilic cytoplasm without cytological atypia. Clear cytoplasm is caused by rich glycogen that is positive for PAS stain. Microcystic SCA has been associated with von Hippel-Lindau gene mutations. The present study reported a classical microcytic serous cystadenoma of the pancreas with atypical clinical manifestations.

\section{Case report}

A 60 year-old female with a history of hypertension and cerebral infarction presented with recurrent bilateral lower back discomfort for 3 months. The lower back discomfort was not associated with jaundice or weight loss. The patient had no significant family history of cancer and had not been previously admitted for these symptoms. An abdominal ultrasonography revealed multiple pancreatic cysts and liver cysts. Serum amylase and cancer biomarkers, including cancer antigen (CA)19-9, carcinoembryonic antigen (CEA) and CA125, were within normal range. A multiphasic contrast-enhanced computed tomography (CT) scan revealed a $4.8 \mathrm{~cm}$ cystic solid mass with unequal moderate enhancement and multiple calcification foci in the pancreatic body (Fig. 1A). Furthermore, magnetic resonance imaging (MRI) revealed a similar cystic solid mass in the pancreatic body without dilatation of the pancreatic duct (Fig. 1B). Combined with the imaging data, a clinical impression of solid-pseudopapillary neoplasms was created. An abdominal exploration was performed and revealed splenic vessels encased by the mass, similar to invasion observed in malignant tumors. Distal pancreatectomy with splenectomy was subsequently performed. The patient's postoperative course was uneventful and she was discharged 15 days after the surgery. Grossly, the tumor was firm and consisted of numerous small cysts on cut surface with a central fibrotic scar. Histopathological examination of the cystic solid mass revealed classical features of microcytic serous cystadenoma of the pancreas with septa centrally coalesced into a 'stellate scar' with calcification and encasing the splenic vessels (Fig. 1C and D).

\section{Discussion}

Serous cystadenoma of the pancreas was first described by Compagno and Oertel in 1978 (1). Morphological features 

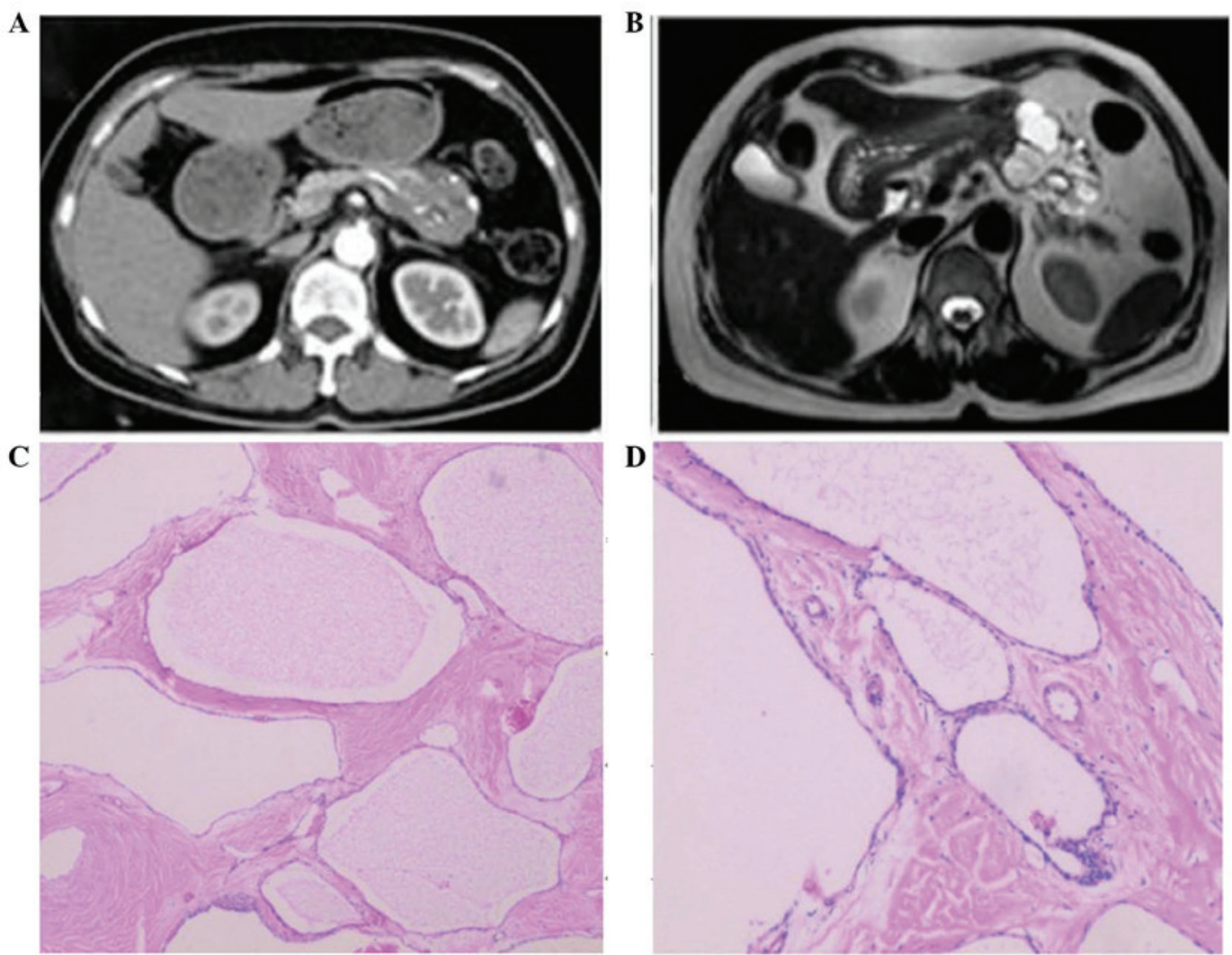

Figure 1. (A) A multiphasic contrast-enhanced computed tomography scan revealed a 4.8 cm cystic solid mass with unequal moderate enhancement and multiple calcification foci in the pancreatic body. (B) Magnetic resonance imaging showed similar cystic solid mass in the pancreatic body without dilatation of the pancreatic duct. The morphology of microcytic serous cystadenoma of the pancreas was assessed by hematoxylin and eosin staining at a magnification of (C) $\mathrm{x} 40$ and (D) $\mathrm{x} 100$

include numerous cysts with variable diameter lined by a single layer of cuboidal epithelium producing watery fluid similar to serum. SCA occurs most frequently in females ranging between 56 and 62 years of age $(4,5)$. SCA can occur in any section of the pancreas, although predominantly in the head and body region (6). SCA is associated with von Hippel-Lindau disease and can have diffuse and multiple lesions over the entire pancreas at a younger age in this setting (7). CT and MRI are the most common diagnostic methods for SCA. CT and MRI imaging of SCA typically reveals a lobulated contour, uneven low density, thin wall/capsule and absence of wall enhancement. Atypical imaging features include multiple septations, wall enhancement and/or calcification, wall thickness, and presence of mural nodules or papillary projections (2). However, these features cannot differentiate SCA from other pancreatic cystic tumors. Approximately $60 \%$ of the SCAs can be diagnosed by $\mathrm{CT}$ and/or MRI. When imaging features reported by $\mathrm{CT}$ and/or MRI are insufficient to differentiate cystic lesions, endoscopic ultrasonography (EUS) with or without EUS-guided fine-needle aspiration (FNA) can provide additional and useful information, and notably improves the sensitivity of diagnosis for SCA to $76 \%$ (8). Although EUS with FNA increases the detection rate of SCA, FNA is somehow limited due to a high rate of insufficient sampling and potential tumor seeding of the needle tract for cases when malignancy cannot be ruled out. Serum CEA and CA19.9 are non-specific biomarkers for pancreatic lesions.
Almost all SCAs are benign and to date, no patient has succumbed to SCA or associated reasons $(9,10)$. Therefore, surgical resection for serous cystic neoplasms should be limited to those with clinical symptoms and large tumor size. A previous study indicated that a tumor $>6 \mathrm{~cm}$ is the cornerstone for operation (11). Based on our experience, the present study suggested that asymptomatic patients with a small lesion $(<4 \mathrm{~cm})$ require imaging surveillance every 2 years. However, tumors $>4 \mathrm{~cm}$ require surgical resection, since these tumors are more frequently symptomatic and faster growing (12). When multiphasic contrast enhanced CT scan and MRI imaging studies reveal a low diagnostic power to differentiate SCA from mucinous cystic neoplasm and intraductal papillary mucinous neoplasm, early surgical treatment must be taken into account with growing experience and advanced surgical techniques.

Surgical resection is considered curative for SCA. The location of SCA in the pancreas determines the method of resection, which is used. The appropriate methods for this case include distal pancreatectomy with spleen-preserving or with splenectomy. Spleen-preserving distal pancreatectomy includes the Warshaw procedure, which conserves the spleen by blood flow from the short gastric vessels and the Kimura procedure, which preserves the spleen with splenic vessels (13). Since the Warshaw procedure has a high incidence of complications and subsequent splenectomies, distal pancreatectomy with splenectomy is often required, particularly when the splenic vessels are encased by SCA, as in this 
case. Therefore, when no recurrence is observed following radical surgery, post-operation follow-up is unnecessary in these patients.

In the previous decade, non-surgical management for patients with SCA is rising. EUS-guided treatment has been reported, particularly for patients unfit for surgery. Cyst ablation with the injection of ethanol followed by paclitaxel was considered as another promising technique and has revealed complete resolution in certain patients, although long-term follow-up is still required $(14,15)$.

In conclusion, with the most recent imaging techniques, a large proportion of patients with SCA cannot be definitively diagnosed prior to operation, particularly with atypical imaging findings. Although SCA is a benign pancreatic cystic neoplasm, the patient can be symptomatic, particularly when the cyst is $>4 \mathrm{~cm}$ and surgical treatment is particularly important to manage these patients.

\section{References}

1. Compagno $\mathrm{J}$ and Oertel JE: Microcystic adenomas of the pancreas (glycogen-rich cystadenomas): A clinicopathologic study of 34 cases. Am J Clin Pathol 69: 289-298, 1978.

2. Choi JY, Kim MJ, Lee JY, Lim JS, Chung JJ, Kim KW and Yoo HS: Typical and atypical manifestations of serous cystadenoma of the pancreas: Imaging findings with pathologic correlation. AJR Am J Roentgenol 193: 136-142, 2009.

3. Kehagias D, Smyrniotis V, Gouliamos A and Vlahos L: Cystic pancreatic neoplasms: Computed tomography and magnetic resonance imaging findings. Int J Pancreatol 28: 223-230, 2000.

4. Galanis C, Zamani A, Cameron JL, Campbell KA, Lillemoe KD, Caparrelli D, Chang D, Hruban RH and Yeo CJ: Resected serous cystic neoplasms of the pancreas: A review of 158 patients with recommendations for treatment. J Gastrointest Surg 11: 820-826, 2007.
5. Bassi C, Salvia R, Molinari E, Biasutti C, Falconi M and Pederzoli P: Management of 100 consecutive cases of pancreatic serous cystadenoma: Wait for symptoms and see at imaging or vice versa? World J Surg 27: 319-323, 2003.

6. Kimura W, Moriya T, Hirai I, Hanada K, Abe H, Yanagisawa A, Fukushima N, Ohike N, Shimizu M, Hatori T, et al: Multicenter study of serous cystic neoplasm of the Japan pancreas society. Pancreas 41: 380-387, 2012.

7. Charlesworth M, Verbeke CS, Falk GA, Walsh M, Smith AM and Morris-Stiff G: Pancreatic lesions in von hippel-lindau disease? A systematic review and meta-synthesis of the literature. J Gastrointest Surg 16: 1422-1428, 2012.

8. Khashab MA, Kim K, Lennon AM, Shin EJ, Tignor AS, Amateau SK, Singh VK, Wolfgang CL, Hruban RH and Canto MI: Should we do EUS/FNA on patients with pancreatic cysts? The incremental diagnostic yield of EUS over CT/MRI for prediction of cystic neoplasms. Pancreas 42: 717-721, 2013.

9. Malleo G, Bassi C and Salvia R: Appraisal of the surgical management for pancreatic serous cystic neoplasms. Surg Endosc 27: 2646-2647, 2013.

10. Abe H, Kubota K, Mori M, Miki K, Minagawa M, Noie T, Kimura W and Makuuchi M: Serous cystadenoma of the pancreas with invasive growth: Benign or malignant? Am J Gastroenterol 93: 1963-1966, 1998.

11. Pravisani R, Intini SG, Girometti R, Avellini C, Leo CA, Bugiantella W and Risaliti A: Macrocystic serous cystadenoma of the pancreas: Report of 4 cases. Int J Surg 21: S98-S101, 2015.

12. Tseng JF, Warshaw AL, Sahani DV, Lauwers GY, Rattner DW and Fernandez-del Castillo C: Serous cystadenoma of the pancreas: Tumor growth rates and recommendations for treatment. Ann Surg 242: 413-419, 2005.

13. Dina I, Ginghina O, Iacobescu C, Vrabie C, Gidea C, Munteanu R, Iosifescu R and Iordache N: Spleen preservation in a caudal pancreatic serous cystadenoma-case report. J Med Life 8: 106-108, 2015.

14. Oh HC, Seo DW, Kim SH, Min B and Kim J: Systemic effect of endoscopic ultrasonography-guided pancreatic cyst ablation with ethanol and paclitaxel. Dig Dis Sci 59: 1573-1577, 2014.

15. Oh HC, Seo DW, Song TJ, Moon SH, Park do H, Soo Lee S, Lee SK, Kim MH and Kim J: Endoscopic ultrasonography-guided ethanol lavage with paclitaxel injection treats patients with pancreatic cysts. Gastroenterology 140: 172-179, 2011. 